\title{
Downregulation of AKT reverses platinum resistance of human ovarian cancers in vitro
}

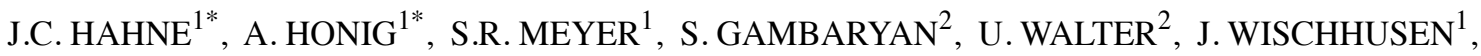 \\ S.F.M. HÄUSSLER ${ }^{1}$, S.E. SEGERER ${ }^{1}$, N. FUJITA ${ }^{3}$, J. DIETL ${ }^{1}$ and J.B. ENGEL ${ }^{4}$ \\ ${ }^{1}$ Department of Gynecology, University of Würzburg, Josef-Schneider-Str. $4 ;{ }^{2}$ Institute for Clinical Biochemistry \\ and Pathobiochemistry, University of Wuerzburg, Grombühlstr. 12, D-97080 Würzburg, Germany; \\ ${ }^{3}$ The Cancer Chemotherapy Center, Japanese Foundation for Cancer Research, Tokyo 135-8550, Japan; \\ ${ }^{4}$ Department of Gynecology, University of Regensburg, Landshuter Str. 65, D-93055 Regensburg, Germany
}

Received April 11, 2012; Accepted May 24, 2012

DOI: 10.3892/or.2012.2041

\begin{abstract}
Platinum resistance is the most crucial problem for treatment of ovarian cancer. Increasing evidence points towards AKT overexpression as a mechanistic reason for this clinical condition. The present study evaluates the effect of overexpression and downregulation of AKT on the sensitivity to cisplatin in a platinum-resistant human ovarian cancer cell line and the corresponding platinum-sensitive parental cell line. A2780 and A2780cis ovarian cancer cell lines were stably transfected with an AKT-sense and AKT-antisense plasmid. Successful transfection was evaluated by western blot analysis. Cytotoxic effects of cisplatin were evaluated by metabolic (MTT) and clonogenicity assays as well as by FACS analysis. AKT overexpression (confirmed by western blotting) converted platinum-sensitive A2780 into platinumresistant cells as shown by MTT assay. Importantly, platinum resistance of A2780cis cells could be reversed by downregulation of AKT, as demonstrated by MTT and clonogenicity assays and FACS analysis. Our data provide strong evidence that cisplatin resistance in ovarian cancer is mediated by AKT overexpression and can be overcome by AKT downregulation, thus, providing a rationale for clinical phase II/III studies combining AKT inhibitors with cisplatin.
\end{abstract}

\section{Introduction}

Ovarian cancer is the most frequent cause of death from gynaecological cancer in western countries (1). At diagnosis, epithelial ovarian cancer is currently treated by cytoreductive surgery followed by platinum-based chemotherapy. While treatment of ovarian cancer with platinum-based agents has

Correspondence to: Dr J.B. Engel, Department of Gynecology, University of Regensburg, Landshuter Str. 65, D-93055 Regensburg, Germany

E-mail: joergbengel@hotmail.com

*Contributed equally

Key words: ovarian cancer, platinum-resistance, AKT been established for decades, these still remain the most active substances for this entity (2). Accordingly, primary resistance to platinum-based adjuvant therapy is associated with a worse disease-free and overall survival (3). However, virtually all patients eventually develop secondary resistance to platinum based agents and compounds used for second or third line treatment display substantially less anti-cancer activity as compared to platinum (4). Thus, there is a definite need for new treatment strategies which overcome platinum-resistance. This would have an immediate impact on the clinical management of patients with advanced ovarian cancer. Accordingly, a better understanding of mechanisms responsible for platinumresistance is of paramount importance.

The serine/threonine kinase AKT/PKB-pathway is a promising target for cancer therapy, as it is a main nodal point where extracellular and intracellular oncogenic signals are integrated. Alterations of the AKT-pathway have been detected in several human malignancies including ovarian cancer (5). AKT has a broad range of downstream effectors that regulate cell processes such as cell growth, cell cycle progression, survival, migration, and angiogenesis (6). Due to the key role of AKT in malignant transformation numerous inhibitors of the AKT-pathway have been developed, and are currently in various stages of clinical development (7).

In human specimens of ovarian cancer AKT was found to be activated in $68 \%$ (5) and PI3K, an upstream component of the AKT-pathway, was found to be mutated in $12 \%$ of the cases (8). Recent evidence by our group and others has shown that overactivation of the AKT-pathway may be associated with platinum-resistance (9-12). The present study evaluates the effect of overexpression and downregulation of AKT on sensitivity to cisplatin in a platinum-sensitive parental human ovarian cancer cell line and the corresponding platinumresistant cell line.

\section{Materials and methods}

Plasmid construction. The plasmid pFLAG-CMV-2-humanWT-Akt1 $(13,14)$ was kindly provided by Dr Naoya Fujita. The DNA-sequence coding for human Akt-1 was amplified by PCR using pFLAG-CMV-2-human-WT-Akt1 as template with 


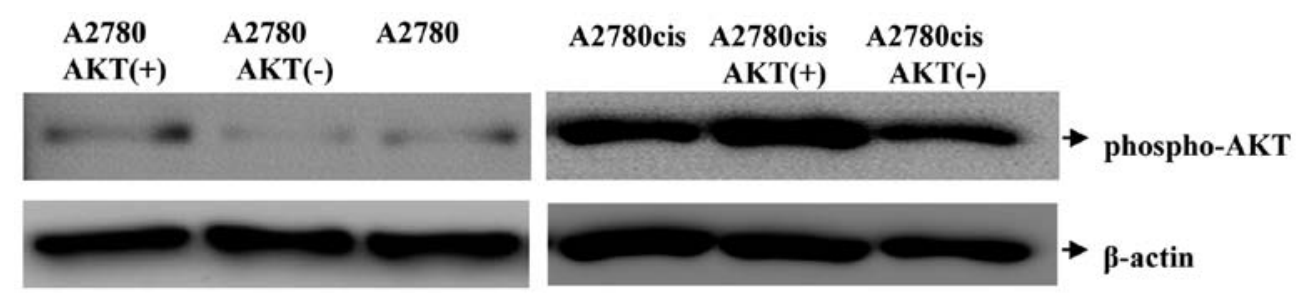

Figure 1. Western blotting to show expression of phosphorylated AKT-1 (phospho-AKT) protein and expression of the housekeeping protein $\beta$-actin.

the following primers: 5'-CGGTGGGAGGATCCTATAAGC AGAGC-3' and 5'-CCATCCCTCCAAGATATCGTC-3'. The resulting PCR product was purified with QIAquick PCR purification kit (Quiagen, Hilden, Germany) and than digested with BamHI and EcoRV (New England Biolabs, Frankfurt, Germany). The Akt-1 coding sequence was cloned into BamHI and $E c o$ RV digested pcDNA(+) and pcDNA(-) (Invitrogen, Karlsruhe, Germany), respectively, resulting in plasmids coding for the Akt-1 sense $\left(\mathrm{AKT}^{+}\right)$or antisense (AKT) cDNA. For verification the resulting plasmids were sequenced (Entelechon, Regensburg, Germany).

Cell culture and stable transfection. A2780 and A2780cis cell lines (both are p53 and KRAS wild-type cell lines) were obtained from ECACC (Salisbury, UK). The cisplatin-resistant A2780cis cell line has been developed by chronic exposure of the parental cisplatin-sensitive A2780 cell line to increasing concentrations of cisplatin (15). Cells were cultured in RPMI, supplemented with $10 \%$ FCS, $100 \mathrm{U}$ per ml penicillin G, and $100 \mathrm{mg} / \mathrm{ml}$ streptomycin (Invitrogen). Transfection of A2780 and A2780cis cells was performed with TransIT-LT1 (Mirus, Madison, WI, USA) according to the manufacturer's instructions. Briefly, $24 \mathrm{~h}$ after seeding cells in 6-well plates the cells were washed with PBS and $1000 \mu$ l serum-free medium was added to each well. Then, in each well a mixture of $100 \mu \mathrm{l}$ serum-free medium, $3 \mu \mathrm{l}$ transfection reagent and $1 \mu \mathrm{g}$ plasmid was added. After 6-h transfection was stopped by replacing the transfection medium with serum-containing medium. Fortyeight hours after transfection selection of transfected cells was started with $200 \mu \mathrm{g} \mathrm{G}-418 / \mathrm{ml}$ (Invitrogen).

Preparation of cell lysates and western blotting. Preparation of cell lysates was performed as previously described (16). Membranes were probed overnight with: anti-phospho-Akt (no. 2118), antibody from Epitomics (Burlingame, CA, USA). The secondary antibody horseradish peroxidase (HRP)conjugated anti-rabbit IgG was from Cell Signaling (Frankfurt, Germany). The chemiluminescent HRP substrate solution (Millipore, Schwalbach, Germany) was used for detection.

MTT assay. To quantify the cytotoxicity of cis-diamminedichloroplatinum(II) (cisplatin) viability of cells was measured with a non-radioactive cell counting assay as previously described (17). Experiments were performed 6-fold, and at least three independent experiments were performed for each cell line.

Clonogenicity assay. Cells were cultured in 96-well flat-bottom plates, in humidified $37^{\circ} \mathrm{C}$ and $5 \% \mathrm{CO}_{2}$ atmosphere. Cell density was initially adjusted to $2.5 \times 10^{3}$ cells $/ \mathrm{ml}$ in a final volume of
$200 \mu 1 /$ well. Twenty-four hours after seeding cells were treated with different concentrations of cisplatin as indicated and the 96-well plates were incubated for 7 days. Then the supernatant was discarded and the cells were washed with PBS before $50 \mu \mathrm{l}$ crystal violet solution $[0.5 \%$ (w/v) in methanol (Roth, Karlsruhe, Germany)] was added in each well. Plates were incubated under shaking for $10 \mathrm{~min}$ at room temperature. After washing three times with tap water the plates were air dried at room temperature. Formed cell colonies were counted under a microscope (Leica DMIL, Wetzla, Germany). Three independent experiments were performed, and each experiment was carried out in triplicate.

Flow cytometry. For cell cycle analysis, cells were treated with cisplatin as indicated, harvested, fixed and permeabilized overnight in ice-cold 70\% ethanol (Merck, Darmstadt, Germany). The cells were washed twice with PBS. RNA was digested with RNase A (Invitrogen). DNA was stained with PI $(50 \mu \mathrm{g} /$ $\mathrm{ml}$ ). Fluorescence was recorded in a FACSCalibur (BectonDickinson, Heidelberg, Germany). Instrument settings were adjusted to move the $G_{0} / G_{1}$ peak to 200 relative fluorescence units. Cells to the left of this peak have a DNA content $<2 n$, indicative of cell death. Aggregated cells were gated out. A total of 20,000 events per condition were recorded.

\section{Results}

Generation of cell lines with stable AKT-overexpression and downregulation. Four stable ovarian cell cultures were established by transfection of platinum-resistant A2780cis and parental A2780 cells with either an AKT-1 or an AKT-1-antisense expression vector. AKT-1-overexpressing (A2780AKT ${ }^{+}$and $\mathrm{A} 2780 \mathrm{cis}^{-\mathrm{AKT}^{+}}$) and underexpressing (A2780AKT ${ }^{-}$and A2780cisAKT) cells were selected from pooled populations of transfected cells, in order to avoid clonal variations. First of all, the established cell lines and the parental cells were analysed on protein level by western blotting with AKT-1-specific antibody (Fig. 1). The observed AKT-1 expression corresponded to the expected variations: the cell line $\mathrm{A} 2780 \mathrm{AKT}^{+}$and A2780cisAKT ${ }^{+}$expressed phosphorylated AKT-1 (phosphoAKT) at high levels, while cells transfected with the AKT-1 antisense plasmid (A2780AKT, A2780cisAKT) displayed only moderate expression of phospho-AKT (Fig. 1). Expression of phospho-AKT was higher in A2780cis than in A2780 cells, as described previously (9).

Morphological changes are visible especially in cell lines derived from A2780cis cells with Akt-overexpression and downregulation (Fig. 2). AKT-overexpression results in cells $\left(\mathrm{A} 2780 \mathrm{cisAKT} \mathrm{T}^{+}\right)$with more spindle-shaped appearance in 

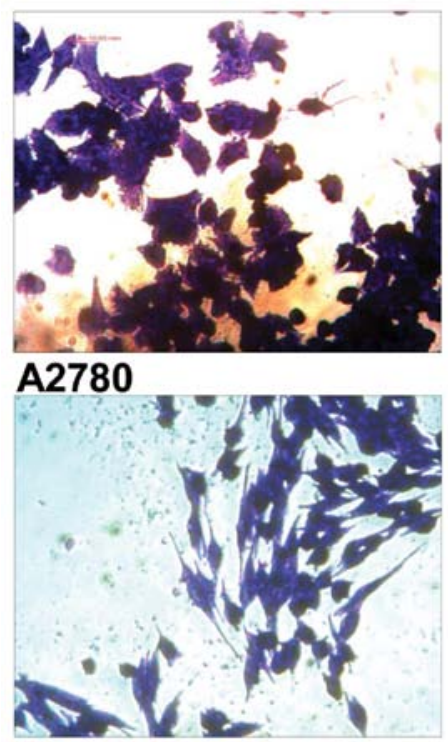

A2780cis AKT+

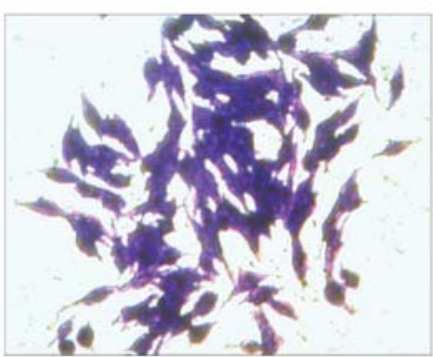

A2780cis

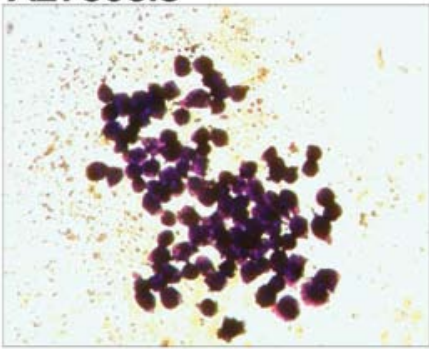

A2780cis AKT-

Figure 2. Typical morphological appearance of the different A2780 cell lines. Cells were stained with crystal violet and pictures were taken at 20-fold initial magnification.

Table I. $\mathrm{IC}_{50}$ of ovarian cancer cell with and without overexpression and downregulation of Akt subsequent to treatment with cisplatin. $^{\mathrm{a}}$

$24 \mathrm{~h}$

\begin{tabular}{|c|c|}
\hline A2780 & $9.4 \pm 0.6$ \\
\hline A2780cis & $>40.0$ \\
\hline A2780 $\mathrm{AKT}^{+}$ & $31.0 \pm 0.3^{\mathrm{a}}$ \\
\hline A2780 AKT - & $7.6 \pm 0.2^{\mathrm{a}}$ \\
\hline $\mathrm{A}^{2780 \mathrm{cis} \mathrm{AKT}^{+}}$ & $>40.0$ \\
\hline A2780cis AKT & $18.9 \pm 0.3^{\mathrm{a}}$ \\
\hline
\end{tabular}

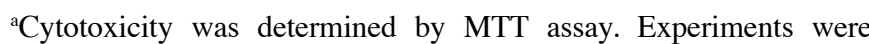

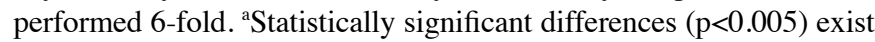
between A2780, A2780 $\mathrm{AKT}^{+}$and A2780 AKT and between A2780cis and A2780cis AKT.

comparison to A2780cis cells. Whereas, AKT-downregulation results in cells (A2780cisAKT) with spheroidal form. In spite of their rounded appearance, cells were viable as determined by MTT and clonogenicity assays.

Cytotoxic effects of cisplatin in ovarian cancer cells with phospho-AKT-overexpression and downregulation. $\mathrm{IC}_{50} \mathrm{~s}$ subsequent to treatment with cisplatin were determined for wildtype A2780 (WT), A2780cis and transfected cells (Table I). As expected, $\mathrm{IC}_{50}$ in WT A2780 cells is significantly lower than in A2780cis cells. Stable transfection with AKT-1 significantly $(\mathrm{p}<0.05)$ increases $\mathrm{IC}_{50}$ in $\mathrm{A} 2780$ after cisplatin addition, but does only slightly increase $\mathrm{IC}_{50}$ in $\mathrm{A} 2780$ cis cells, which are already platinum-resistant. On the other hand, stable transfection with antisense AKT-1 slightly but significantly decreases $\mathrm{IC}_{50}$ in A2780 cells, but significantly $(\mathrm{p}<0.05)$ decreases $\mathrm{IC}_{50}$ in platinum-resistant A2780cis cells. With A2780cis, A2780cisAKT ${ }^{+}$
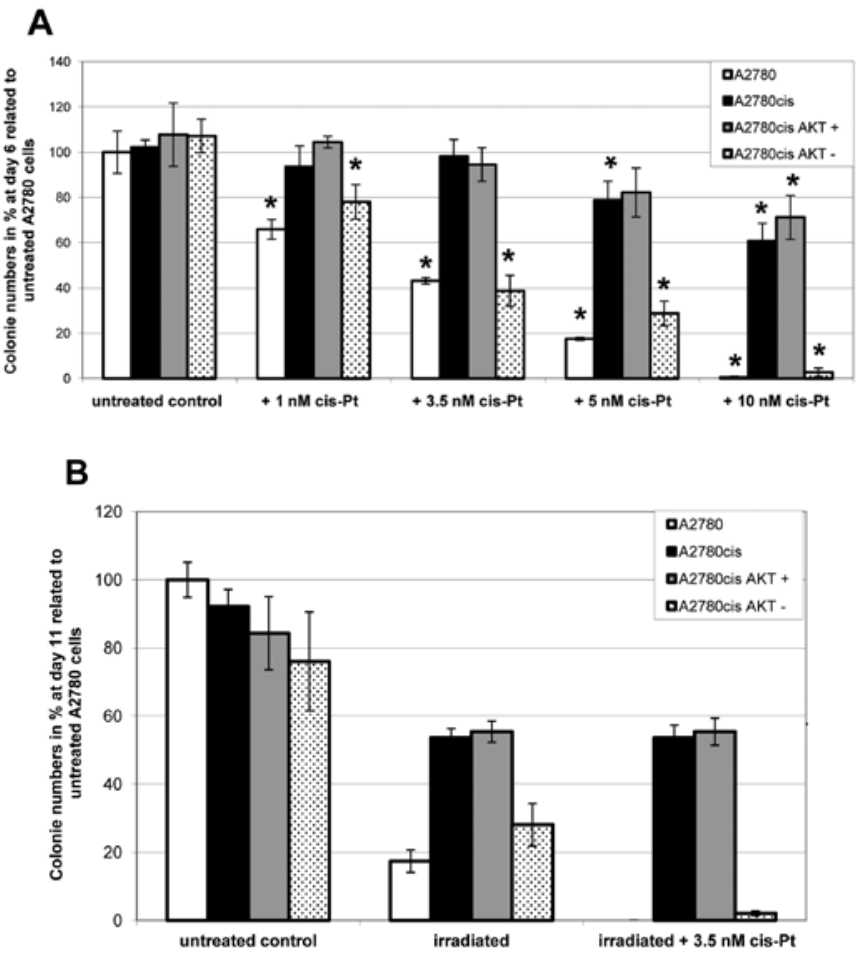

Figure 3. Clonogenicity assays. (A) Cells were treated with different concentrations of cisplatin (cis-Pt) for 6 days and (B) cells were first irradiated with $2.5 \mathrm{~Gy}$ and then treated with $3.5 \mathrm{nM}$ cisplatin (cis-Pt) for 11 days. Cells were stained and fixed with crystal violet. The formed cell colonies were counted. The figure shows the colony numbers in relation to the colonies formed by untreated A2780 (set to 100\%). Three independent experiments were performed, and each experiment was carried out in triplicate. Statistically significant difference $(\mathrm{p}<0.05)$ between a sample and the relevant control is indicated by an asterisk.

and A2780cisAKT cells, similar results were obtained by clonogenicity assays (Fig. 3), confirming our hypothesis that AKT-downregulation can break platinum-resistance in ovarian 
A

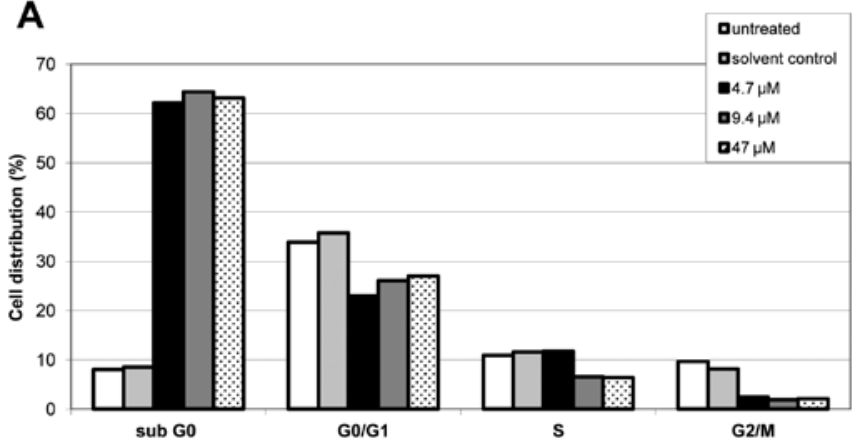

C

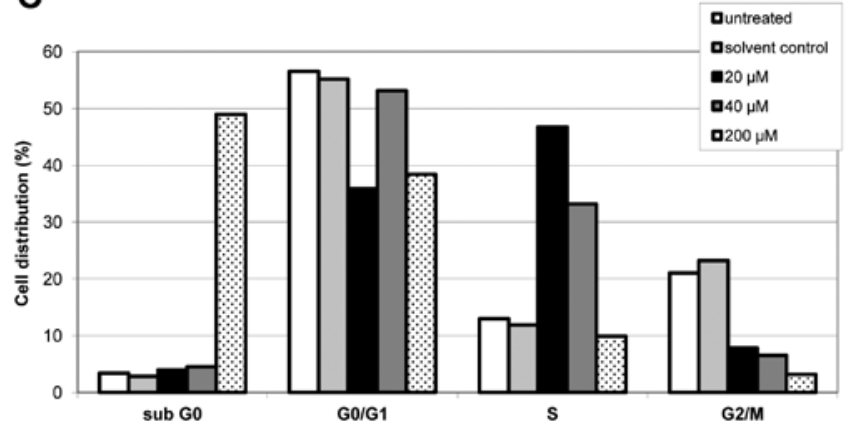

B

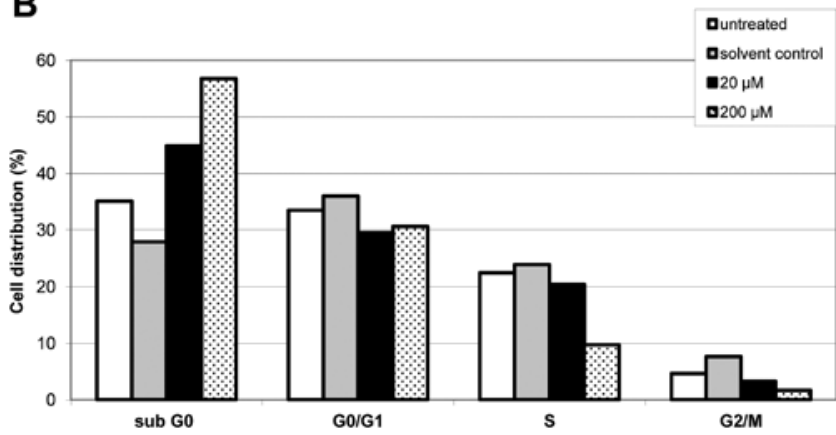

D

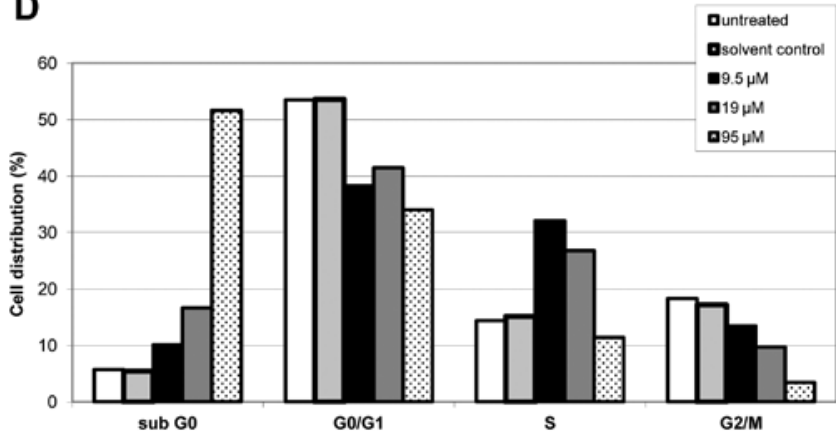

Figure 4. Effect of cisplatin (cis-Pt) on the cell cycle distribution. A2780 (A), A2780cis (B), A2780cis AKT ${ }^{+}$(C) and A2780cis AKT (D) cells were treated with different concentrations of cisplatin (cis-Pt) for $24 \mathrm{~h}$, fixed, permeabilzed, stained with propidium iodide and analysed by flow cytometry. The figure shows the distribution of the cells to the different phases of cell cycle (\%).

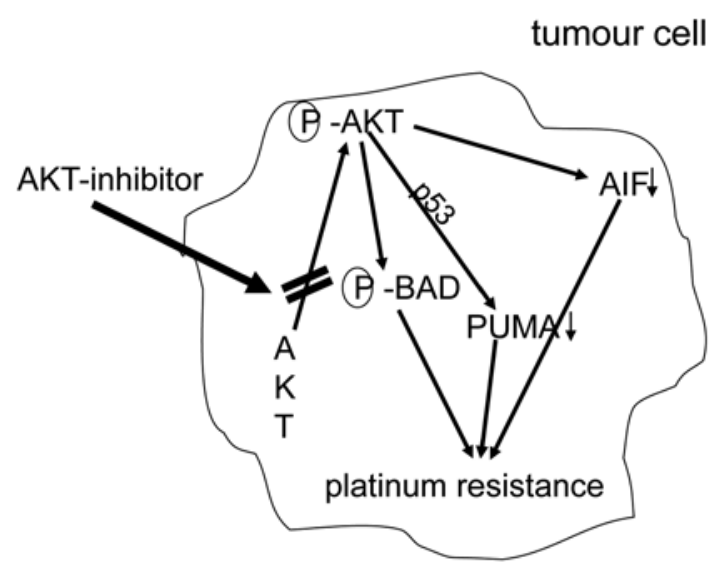

Figure 5. AKT phosphorylation mediates phosphorylation of BAD, upregulation of PUMA and downregulation of apoptosis inhibitory factor (AIF), thus inducing resistance to apoptosis and resistance to platinum compounds.

cancer cells. In untreated controls all cell lines exhibit the same growing capacity. After addition of increasing amounts of cisplatin, however, colony formation differs significantly between the cell lines; nevertheless all cell lines show a dosedependent decrease in growing capacity. Parental A2780 and A2780cisAKT cells cannot grow in the presence of $10 \mathrm{nM}$ cisplatin. In contrast, A2780cis and A2780cisAKT ${ }^{+}$cells are still growing in the presence of $10 \mathrm{nM}$ cisplatin, forming between 60 and $70 \%$ of colonies compared to untreated controls.

Irradiation with 2.5 Gy results in reduced colony formation in all cell lines. Once again the growing capacity of A2780cisAKT cells is comparable to parental A2780 cells. Combination of irradiation with $3.5 \mathrm{nM}$ cisplatin inhibits the growth of WT A2780 and A2780cisAKT cells completely. This combination treatment decreases colony formation only $40 \%$ in the A2780cis and A2780cisAKT ${ }^{+}$cells.

Effects of cisplatin on cell cycle distribution in ovarian cancer cells with phospho-AKT-overexpression and downregulation. Effects of different doses of cisplatin in A2780cis, $\mathrm{A} 2780$ cis $\mathrm{AKT}^{+}$and $\mathrm{A} 2780$ cisAKT on cell cycle distribution were determined by FACS analysis. Cisplatin induced apoptosis in all phases of the cell cycle, however, to a greater extent in the $\mathrm{S}$ and the $\mathrm{G}_{2} / \mathrm{M}$ phase. This effect occurred regardless of AKT overexpression or downregulation in A2780cis cells (Fig. 4). FACS analysis also shows that induction of apoptosis (reflected by a dose-dependent increase of the sub- $\mathrm{G}_{0}$ fraction) occurs at substantially lower doses of cisplatin in A2780cisAKT, than in A2780cisAKT ${ }^{+}$and A2780cis cells, which is in good accordance with the results of our cytoxicity and clonogenic assays.

\section{Discussion}

The effect of the phosphatidylinositol-3-kinase (PI-3K)/AKT cascade on proapoptotic protein BAD, a known substrate of AKT, has been studied in both cisplatin-resistant Caov-3 and -sensitive A2780 human ovarian cancer cells (18). Treatment of Caov-3 and A2780 cells with cisplatin stimulated the activation of AKT, and the PI-3K inhibitor wortmannin blocked the cisplatin-induced AKT-activation. Cisplatin treatment also stimulated phosphorylation of BAD at the Ser-112 and Ser-136 sites in Caov-3 and A2780 cells. Whereas phosphorylation of BAD at Ser-136 was blocked by treatment with wortmannin, its phosphorylation at Ser-112 was blocked by a MAP/ERK kinase 
inhibitor, PD98059. Exogenous transient expression of a dominant-negative AKT in both Caov-3 and A2780 cells decreased cell viability after treatment with cisplatin. In contrast, no sensitization to cisplatin was observed in cells expressing wildtype AKT. These findings suggested that cisplatin-induced DNA damage causes the phosphorylation of BAD via an extracellular signal-regulated protein kinase (ERK) cascade and via a PI-3K/AKT cascade. Inhibition of either of these cascades sensitizes ovarian cancer cells to cisplatin, thus providing the first evidence, that the AKT-pathway is involved in cisplatinresistance in ovarian cancers (18). Additional results suggest that AKT confers platinum-resistance, in part, by modulating the direction of $\mathrm{p} 53$ on the caspase-dependent mitochondrial death pathway (19). Thus, in ovarian cancers p53 is a determinant of platinum sensitivity and AKT contributes to chemoresistance, in part, by attenuating p53-mediated PUMA upregulation and phosphorylation of p53 (20). Recent results suggest that in platinum sensitive ovarian cancer cells, cisplatin-induced apoptosis can also proceed, in part, via a caspase-independent mechanism involving apoptosis inducing factor (AIF), and that AKT activation additionally confers resistance to cisplatininduced apoptosis by blocking this pathway (21).

The anti-tumor effect of tangeretin, a citrus flavonoid known to inhibit cancer cell proliferation, was investigated in combination with cisplatin in in vitro models of A2780/CP70 and 2008/ $\mathrm{C} 13$ cisplatin-resistant human ovarian cancers (22). Pretreatment of cells with tangeretin before cisplatin treatment synergistically inhibited cancer cell proliferation. Interestingly, phospho-AKT and its downstream substrates, e.g., NF- $\kappa$ B, phospho-GSK-3 $\beta$, and phospho-BAD, were downregulated upon tangeretincisplatin treatment. The tangeretin-cisplatin-induced apoptosis in A2780/CP70 cells was increased by PI3K inhibition and siRNA-mediated AKT silencing, but reduced by overexpression of constitutively activated AKT. Although the overall results can only be interpreted with caution, as natural compounds such as tangeretin may display different effects apart from AKT-inhbition, tangeretin exposure preconditions cisplatinresistant human ovarian cancer cells for cisplatin-induced cell death. This effect may occur through downregulation of the PI3K/AKT signaling pathway.

In addition to these in vitro studies, in a series of 98 patients with amplification of PI3K, an upstream component of the AKT-pathway was associated with resistance to platinum-based chemotherapy (23). Accordingly, Woenckhaus et al found that PIK3CA amplification was a strong predictor for early tumorassociated death in ovarian cancer patients (24).

Recent work by our group evaluated the anti-tumor efficacy of the AKT inhibitor perifosine in platinum-sensitive and -resistant human ovarian cancer cells (9). We used the platinum-sensitive A2780 cell line and the A2780cis cell line, which displays secondary resistance to cisplatin and was generated from the parental A2780 cells by exposure to increasing doses of cisplatin (15). Thus, in two cell lines with the same genetic background we could show that A2780cis cells express substantially higher levels of phospho-AKT and are more sensitive to treatment with AKT-inhibitor perifosin. Furthermore, coincubation with perifosine sensitized A2780cis cells to treatment with cisplatin. AKT-inhibitor perifosine has been tested in phase II studies in patients with breast, prostate, pancreatic, head and neck cancer, malignant melanoma, multiple myeloma, colorectal cancer and soft tissue sarcoma (25-31). A recent phase I study with perifosine combined with radiotherapy performed in patients with advanced solid tumors has shown preliminary evidence of anti-cancer activity, including complete responses (32). Thus, perifosine is an attractive compound for further clinical studies in tumor entities, such as platinum-resistant ovarian cancers.

The clinical observation, that the AKT-inhibitor perifosine displays synergy with radiotherapy is in accordance with our results obtained by clonogenicity assays, as the effect of radiation was most pronounced in tumor cells with low or downregulated AKT (e.g., A2780cisAKT cells) (Fig. 3B).

In order to further elucidate the effect of AKT-expression on platinum-resistance, we established four stable ovarian cancer lines by transfection of platinum-resistant A2780cis and parental A2780 cells with either an Akt-1 or an Akt-1-antisense expression vector. Furthermore, in order to avoid clonal variations, cells

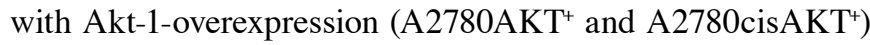
and downregulation (A2780AKT and A2780cisAKT) were selected from pooled populations of transfected cells. Thus, we could show in two cell lines with the same genetic background that AKT-overexpression rendered platinum-sensitive A2780 cells platinum-resistant. Still more importantly, platinum-resistance of A2780cis cells could be reversed by downregulation of AKT. However, AKT-overexpression in platinum-resistant A2780cis cells did not increase platinum-resistance any further. These effects were shown by MTT-assay as well as by clonogenicity assay and FACS analysis. Additionally, we could show by FACS that cisplatin induces cell cycle arrest predominantly in the $\mathrm{S}$ and the G2/M phase but also in the G1 phase regardless of the AKT-expression status, as previously described (33). However, required doses of cisplatin were substantially higher in cell lines with AKT-overexpression. Fig. 5 summarizes mechanisms of platinum-resistance due to overxpression of AKT.

In conclusion, the present study provides strong evidence that in ovarian cancers resistance to cisplatin is mediated by AKT-overexpression and can be overcome by AKT-downregulation. In context with the already existing evidence, our data could be a rationale for phase II/III studies in patients with advanced platinum-resistant ovarian cancers with AKT-inhbitors, such as perifosine and cisplatin.

\section{Acknowledgements}

We appreciate the permission to use the INTAS ChemoStar Imager (Department of Microbiology, University of Würzburg). Therefore we thank especially Professor T. Rudel and Dr B. Bergmann. This work was supported by IZKF Würzburg.

\section{References}

1. Jemal A, Siegel R, Xu J and Ward E: Cancer statistics, 2010. CA Cancer J Clin 60: 277-300, 2010.

2. Pignata S, Cannella L, Leopardo D, Pisano C, Bruni GS and Facchini G: Chemotherapy in epithelial ovarian cancer. Cancer Lett 303: 73-83, 2011.

3. Gonzalez-Martin AJ: Medical treatment of epithelial ovarian cancer. Expert Rev Anticancer Ther 4: 1125-1143, 2004.

4. Matsuo K, Lin YG, Roman LD and Sood AK: Overcoming platinum resistance in ovarian carcinoma. Expert Opin Investig Drugs 19: 1339-1354, 2010.

5. Altomare DA, Wang HQ, Skele KL, et al: AKT and mTOR phosphorylation is frequently detected in ovarian cancer and can be targeted to disrupt ovarian tumor cell growth. Oncogene 23: 5853-5857, 2004. 
6. Cheng JQ, Lindsley CW, Cheng GZ, Yang H and Nicosia SV: The Akt/PKB pathway: molecular target for cancer drug discovery. Oncogene 24: 7482-7492, 2005.

7. Steelman LS, Chappell WH, Abrams SL, et al: Roles of the Raf/ MEK/ERK and PI3K/PTEN/Akt/mTOR pathways in controlling growth and sensitivity to therapy-implications for cancer and aging. Aging 3: 192-222, 2011.

8. Levine DA, Bogomolniy F, Yee CJ, et al: Frequent mutation of the PIK3CA gene in ovarian and breast cancers. Clin Cancer Res 11: 2875-2878, 2005.

9. Engel JB, Schonhals T, Hausler S, et al: Induction of programmed cell death by inhibition of AKT with the alkylphosphocholine perifosine in in vitro models of platinum sensitive and resistant ovarian cancers. Arch Gynecol Obstet 283: 603-610, 2011.

10. Santiskulvong C, Konecny GE, Fekete M, et al: Dual targeting of phosphoinositide 3-kinase and mammalian target of rapamycin using NVP-BEZ235 as a novel therapeutic approach in human ovarian carcinoma. Clin Cancer Res 17: 2373-2384, 2011.

11. Westfall SD and Skinner MK: Inhibition of phosphatidylinositol 3-kinase sensitizes ovarian cancer cells to carboplatin and allows adjunct chemotherapy treatment. Mol Cancer Ther 4: 1764-1771, 2005.

12. Benedetti V, Perego P, Luca Beretta G, et al: Modulation of survival pathways in ovarian carcinoma cell lines resistant to platinum compounds. Mol Cancer Ther 7: 679-687, 2008

13. Sato S, Fujita $\mathrm{N}$ and Tsuruo T: Modulation of Akt kinase activity by binding to Hsp90. Proc Natl Acad Sci USA 97: 10832-10837, 2000.

14. Katayama K, Fujita $\mathrm{N}$ and Tsuruo T: Akt/protein kinase $\mathrm{B}$-dependent phosphorylation and inactivation of WEE1Hu promote cell cycle progression at $\mathrm{G} 2 / \mathrm{M}$ transition. Mol Cell Biol 25: 5725-5737, 2005.

15. Behrens BC, Hamilton TC, Masuda $\mathrm{H}$ et al: Characterization of a cis-diamminedichloroplatinum(II)-resistant human ovarian cancer cell line and its use in evaluation of platinum analogues. Cancer Res 47: 414-418, 1987.

16. Engels K, Knauer SK, Loibl S, et al: NO signaling confers cytoprotectivity through the survivin network in ovarian carcinomas. Cancer Res 68: 5159-5166, 2008.

17. Campling PJ, Galbraith PR and Cole SP: Use of the MTT assay for rapid determinarion of chemosensitivity of human leukemic blast cells. Leuk Res 12: 823-831, 1988.

18. Hayakawa J, Ohmichi M, Kurachi H, et al: Inhibition of BAD phosphorylation either at serine 112 via extracellular signal-regulated protein kinase cascade or at serine 136 via Akt cascade sensitizes human ovarian cancer cells to cisplatin. Cancer Res 60: 5988-5994, 2000.

19. Yang X,Fraser M,Moll UM,Basak A and Tsang BK: Akt-mediated cisplatin resistance in ovarian cancer: modulation of p53 action on caspase-dependent mitochondrial death pathway. Cancer Res 66 : $3126-3136,2006$
20. Fraser M, Bai T and Tsang BK: Akt promotes cisplatin resistance in human ovarian cancer cells through inhibition of p53 phosphorylation and nuclear function. Int J Cancer 122: 534-546, 2008

21. Yang X, Fraser M, Abedini MR, Bai T and Tsang BK: Regulation of apoptosis-inducing factor-mediated, cisplatin-induced apoptosis by Akt. Br J Cancer 98: 803-808, 2008.

22. Arafa el SA, Zhu Q, Barakat BM, et al: Tangeretin sensitizes cisplatin-resistant human ovarian cancer cells through downregulation of phosphoinositide 3-kinase/Akt signaling pathway. Cancer Res 69: 8910-8917, 2009.

23. Kolasa IK, Rembiszewska A, Felisiak A, et al: PIK3CA amplification associates with resistance to chemotherapy in ovarian cancer patients. Cancer Biol Ther 8: 21-26, 2009.

24. Woenckhaus J, Steger K, Sturm K, Munstedt K, Franke FE and Fenic I: Prognostic value of PIK3CA and phosphorylated AKT expression in ovarian cancer. Virchows Arch 450: 387-395, 2007.

25. Marsh Rde W, Rocha Lima CM, Levy DE, Mitchell EP, Rowland KM Jr and Benson AB III: A phase II trial of perifosine in locally advanced, unresectable, or metastatic pancreatic adenocarcinoma. Am J Clin Oncol 30: 26-31, 2007.

26. Leighl NB, Dent S, Clemons M, et al: A phase 2 study of perifosine in advanced or metastatic breast cancer. Breast Cancer Res Treat 108: 87-92, 2007.

27. Snyder EL, Bailey D, Shipitsin M, Polyak K and Loda M Identification of CD44v6(+)/CD24- breast carcinoma cells in primary human tumors by quantum dot-conjugated antibodies. Lab Invest 89: 857-866, 2009.

28. Argiris A, Cohen E, Karrison T, et al: A phase II trial of perifosine, an oral alkylphospholipid, in recurrent or metastatic head and neck cancer. Cancer Biol Ther 5: 766-770, 2006.

29. Knowling M, Blackstein M, Tozer R, et al: A phase II study of perifosine (D-21226) in patients with previously untreated metastatic or locally advanced soft tissue sarcoma: a National Cancer Institute of Canada Clinical Trials Group trial. Invest New Drugs 24: 435-439, 2006

30. Posadas EM, Gulley J, Arlen PM, et al: A phase II study of perifosine in androgen independent prostate cancer. Cancer Biol Ther 4: 1133-1137, 2005.

31. Ernst DS, Eisenhauer E, Wainman N, et al: Phase II study of perifosine in previously untreated patients with metastatic melanoma. Invest New Drugs 23: 569-575, 2005.

32. Vink SR, Schellens JH, Beijnen JH, et al: Phase I and pharmacokinetic study of combined treatment with perifosine and radiation in patients with advanced solid tumours. Radiother Oncol 80: 207-213, 2006.

33. He G, Kuang J, Khokhar AR and Siddik ZH: The impact of S- and G2-checkpoint response on the fidelity of G1-arrest by cisplatin and its comparison to a non-cross-resistant platinum(IV) analog. Gynecol Oncol 122: 402-409, 2011. 